\title{
Conformal transformations and strings for an accelerating quark-antiquark pair in $\mathrm{AdS}_{3}$
}

\author{
Shijong Ryang \\ Department of Physics, Kyoto Prefectural University of Medicine, \\ Inamori Memorial Hall, Shimogamo, Kyoto 606-0823, Japan \\ E-mail: ryang@koto.kpu-m.ac.jp
}

ABSTRACT: From a simple moving open string solution dual to a moving heavy quark with constant velocity in the Poincare $A d S_{3}$ spacetime, we construct an accerlerating open string solution dual to a heavy quark-antiquark pair accelerated in opposite directions by performing the three mappings such as the $\mathrm{SL}(2, R)_{L} \times \mathrm{SL}(2, R)_{R}$ isometry transformation, the special conformal transformation and the conformal $\mathrm{SO}(2,2)$ transformation. Using the string sigma model action we construct two open string solutions staying in two different regions whose dividing line is associated with the event horizon appeared on the string worldsheet and obtain the accelerating open string solution by gluing two such solutions.

Keywords: AdS-CFT Correspondence, Bosonic Strings

ARXIV EPRINT: 1412.2428 


\section{Contents}

1 Introduction 1

2 The accelerating string in the Nambu-Goto action 3

3 Conformal transformations 5

4 The accelerating string in the string sigma model action 10

5 Conclusion $\quad 14$

\section{Introduction}

The AdS/CFT correspondence [1-3] has more and more revealed the strong coupling behaviors of the $\mathcal{N}=4$ super Yang-Mills (SYM) theory by using the string theory in $A d S_{5} \times S^{5}$ where various open and closed string solutions are studied.

By using the Nambu-Goto action there have been constructions of the moving open string solutions with constant velocity in $A d S_{5}$ black hole geometries [4, 5], where the dynamics of quark moving in strongly coupled $\mathcal{N}=4 \mathrm{SYM}$ thermal plasma is investigated by regarding the infinitely massive quark as the open string end at the boundary of the $A d S_{5}$-Schwarzschild spacetime [5] and the finitely massive quark as the open string end at the D7-brane [6] in the $A d S_{5}$-Schwarzschild spacetime [4].

Mikhailov has used the Nambu-Goto action in the Poincare $A d S_{5}$ spacetime to present an analytic generic solution for the open string dual to a single infinitely massive quark moving on an arbitrary timelike trajectory in the $\mathcal{N}=4 \mathrm{SYM}$ theory and extract a rate of the energy loss which agrees with the Lienard formula [7]. Based on the extension of this generic solution to the finite quark mass case it has been shown that an event horizon appears on the worldsheet whenever the single quark accelerates in any fashion [8]. The division of an open string through the horizon is associated with two contributions to the energy-momentum of the string corresponding to the intrisic and radiated energymomentum of the quark [8-10].

An accelerating open string solution dual to a heavy quark-antiquark pair uniformly accelerated in opposite directions has been found [11] by using the Nambu-Goto action in the Poincare $A d S_{5}$ spacetime. The event horizon has been shown to appear on the worldsheet of the open string connecting a quark and an antiquark and separate the radiation and the quark. It has been demonstrated that the rate of energy flow across the horizon becomes the same as derived from the Lienard formula of ref. [7]. 
The similar accelerating open string solution has been constructed by analyzing the Nambu-Goto action in the Rindler spacetime which is given by a coordinate transformation from the AdS spacetime [12]. It has been studied that the energy loss via the moving open string in $A d S_{5}$-Schwarzschild spacetime is related with the appearance of the worldsheet horizon [13-15] and the worldsheet Hawking radiation generates the stochastic motion of the quark $[16,17]$.

The accelerating string solution [11] associated with a uniformly accelerating quarkantiquark pair has been constructed $[18,19]$ as a particular instance from the generic string solution [7] dual to a single quark moving on an arbitrary trajectory. The generic string solution has been indirectly shown [7] to extremize the action, and further has been directly substituted into the string equation of motion and confirmed to solve it [20]. There have been various investigations of the thermal effects of the worldsheet horizon on the accelerating string which are associated with the Unruh temperature [18, 21, 22].

Starting from the generic string solution in the Poincare $A d S_{5}$ spacetime [7] and using a suitable coordinate transformation, the accelerating open string solution dual to a single accelerating quark in the global $A d S_{5}$ spacetime has been constructed [23] (see also [24]).

Recently it has been conjectured that the entanglement of the general quantum Einstein-Podolsky-Rosen (EPR) pair is intimately related with the Einstein-Rosen bridge or the non-traversable wormhole [25]. Associated with the existence of horizon on the worldsheet of the accelerating open string dual to a uniformly accelerating quark-antiquark pair, there have been several studies where the quark-antiquark pair is concretely regarded as a color singlet EPR pair in the $\mathcal{N}=4 \mathrm{SYM}$ theory and its entanglement is encoded in a non-traversable wormhole on the worldsheet of the flux tube connecting the pair [26-29]. The entanglement entropy of the quark-antiquark pair has been investigated $[26,30,31]$ and the relation between the entanglement entropy and the string surface describing gluon scattering in position space has been studied [32].

We will use the Nambu-Goto action in the static gauge for the open string in the Poincare $A d S_{3}$ spacetime and make an ansatz for the string profile expressed by three parameters to reconstruct the two string solutions associated with the one-cusp Wilson loop [33] and the accelerating string solution dual to an accelerating quark-antiquark pair [11].

We will consider the $\mathrm{SL}(2, R)_{L} \times \mathrm{SL}(2, R)_{R}$ isometry group of the $A d S_{3}$ spacetime $[34,35]$ and make this isometry transformation for a simple moving string solution dual to a moving quark with constant velocity to construct the accelerating string solution dual to a uniformly accelerating quark-antiquark pair. This isomery transformation will be applied further to the accelerating string solution. For the moving string solution with constant velocity and the accelerating string solution we will perform the special conformal transformation in the Poincare coordinates and the conformal $\mathrm{SO}(2,2)$ transformation in the embedding coordinates to see what kinds of string solutions appear.

Based on the string sigma model action we will make a special ansatz for the string profile in the factorized form and construct the accelerating string solution on which the event horizon appears. 


\section{The accelerating string in the Nambu-Goto action}

Based on the Nambu-Goto action we consider an open string in $A d S_{3}$ with the Poincare metric

$$
d s^{2}=\frac{d z^{2}-d t^{2}+d x^{2}}{z^{2}},
$$

where we have set the AdS radius $R$ to unity. We use the static gauge

$$
t=\tau, \quad z=\sigma
$$

to express the string action in the Lorentzian worldsheet coordinates

$$
S=-\frac{\sqrt{\lambda}}{2 \pi} \int d \tau d \sigma \frac{\sqrt{D}}{\sigma^{2}}
$$

with $D=1-\left(\partial_{\tau} x\right)^{2}+\left(\partial_{\sigma} x\right)^{2}$. In the equation of motion for $x$

$$
\partial_{\tau}\left(\frac{\partial_{\tau} x}{\sigma^{2} \sqrt{D}}\right)=\partial_{\sigma}\left(\frac{\partial_{\sigma} x}{\sigma^{2} \sqrt{D}}\right)
$$

we make an ansatz

$$
x= \pm \sqrt{A \tau^{2}+B-C \sigma^{2}}
$$

to have

$$
A \partial_{\tau}\left(\frac{\tau}{\sigma^{2} \sqrt{F}}\right)=-C \partial_{\sigma}\left(\frac{1}{\sigma \sqrt{F}}\right)
$$

with $F=A(1-A) \tau^{2}+B-C(1-C) \sigma^{2}$.

For $B \neq 0$ there is one solution specified by $A=C=1$ which yields the string configuration expressed in terms of $B \equiv b^{2}$ as

$$
x= \pm \sqrt{t^{2}+b^{2}-z^{2}} .
$$

This string solution was found in ref. [11] where the infinitely massive quark and antiquark are located on the hyperbolic trajectories $x= \pm \sqrt{t^{2}+b^{2}}$ at the AdS boundary $z=0$ such that the plus/minus sign of (2.7) represents the right and left half of the accelerating string. The quark and antiquark first approach to each other in decelerating and stop to return back in accelerating away from each other with proper acceleration $1 / b$.

Putting the probe D7-branes at $z=z_{m}$ we introduce the finite mass of quark and antiquark as $m=\sqrt{\lambda} / 2 \pi z_{m}$, where the quark and antiquark are two ends of an open string at $z=z_{m}$. After the replacement $b^{2} \rightarrow b^{2}+z_{m}^{2}$ for $(2.7)[9,10,18]$ we have

$$
x= \pm \sqrt{t^{2}+b^{2}+z_{m}^{2}-z^{2}}
$$

from which the hyperbolic trajectories of the finitely massive quark and antiquark at $z=z_{m}$ are also given by $x= \pm \sqrt{t^{2}+b^{2}}$. The quark and antiquark are accelerated by turning on the constant electric field $E$ on the D7-branes. The electric field $E$ is related with the proper acceleration $1 / b$ as

$$
\frac{1}{b}=\frac{E}{\sqrt{m^{2}-\lambda E^{2} / 4 \pi^{2} m^{2}}}
$$


whose inversion is given by

$$
E=\frac{m}{\sqrt{b^{2}+\lambda / 4 \pi^{2} m^{2}}}
$$

For $B=0$ there are two solutions which are provided by $A \neq 0,1$ with $C=0$ and $C \neq 0,1$ with $A=1$. The former gives a simple solution

$$
x= \pm \sqrt{A} t
$$

while the latter is complementary to (2.7) and leads to a string solution

$$
x= \pm \sqrt{2 C\left(t^{2}-\frac{z^{2}}{2}\right)}
$$

whose $C$ is fixed as $C=A / 2=1 / 2$. The latter string configuration is described by

$$
z=\sqrt{2\left(t^{2}-x^{2}\right)}
$$

which is the one-cusp Wilson loop solution of [33], where the open string surface ends on two semi infinite lightlike lines. This solution yields pure imaginary Lagrangian so that the amplitude shows the exponential suppression. The planar four-gluon scattering amplitude was computed by using the four-cusp Wilson loop solution in the T-dual AdS spacetime which was obtained from the one-cusp Wilson loop solution by perfoming the conformal $\mathrm{SO}(2,4)$ transformation $[36]$.

In [37] the following two-cusp Wilson loop solution was constructed by applying the conformal $\mathrm{SO}(2,4)$ transformation to the one-cusp Wilson loop solution

$$
z^{2}=t^{2}-x^{2} \pm \sqrt{2\left(t^{2}+x^{2}\right)-1}
$$

whose surface ends on four lines $t=x \pm 1, t=-x \pm 1$ which meet at two cusps $(t, x)=$ $(0, \pm 1)$ for the plus sign and two cusps $(t, x)=( \pm 1,0)$ for the minus sign. The appropriate square of (2.14) yields an equation for $x$ in the fourth order whose solution is given by

$$
x^{2}=t^{2}+1-z^{2} \pm \sqrt{4 t^{2}-2 z^{2}}
$$

As the string solution in the Poincare coodinates can be rescaled as $x^{\mu}=(t, x) \rightarrow$ $x^{\mu} / b, z \rightarrow z / b$, the expression (2.15) becomes

$$
x= \pm\left(t^{2}+b^{2}-z^{2} \pm 2 b \sqrt{t^{2}-\frac{z^{2}}{2}}\right)^{1 / 2}
$$

which shows a suggestive expression that contains two polynomials $t^{2}+b^{2}-z^{2}$ and $t^{2}-z^{2} / 2$ of (2.7) and (2.12). The expression (2.16) as a convolution of two square roots is confirmed indeed to solve the string equation of motion (2.4) through $D=-b^{2} \sigma^{2} / 4 x^{2}\left(\tau^{2}-\sigma^{2} / 2\right)$. 


\section{Conformal transformations}

We use $w^{ \pm} \equiv x \pm t$ to rewrite the Poincare metric (2.1) as $d s^{2}=\left(d z^{2}+d w^{+} d w^{-}\right) / z^{2}$. The $\mathrm{SL}(2, R)_{L} \times \mathrm{SL}(2, R)_{R}$ isometry group for this $A d S_{3}$ metric was investigated in ref. [34] (see also [38]). The $\mathrm{SL}(2, R)_{L}$ transformation is given by

$$
\begin{aligned}
w^{+} \rightarrow w^{+^{\prime}} & =\frac{\alpha w^{+}+\beta}{\gamma w^{+}+\delta}, \quad w^{-} \rightarrow w^{-\prime}=w^{-}+\frac{\gamma z^{2}}{\gamma w^{+}+\delta}, \\
z \rightarrow z^{\prime} & =\frac{z}{\gamma w^{+}+\delta}
\end{aligned}
$$

with real $\alpha, \beta, \gamma, \delta$ obeying $\alpha \delta-\beta \gamma=1$, while the $\operatorname{SL}(2, R)_{R}$ transformation is

$$
\begin{aligned}
w^{+} \rightarrow w^{+^{\prime}} & =w^{+}+\frac{\gamma z^{2}}{\gamma w^{-}+\delta}, \quad w^{-} \rightarrow w^{-^{\prime}}=\frac{\alpha w^{-}+\beta}{\gamma w^{-}+\delta}, \\
z \rightarrow z^{\prime} & =\frac{z}{\gamma w^{-}+\delta} .
\end{aligned}
$$

In view of (3.1) and (3.2) $\alpha$ and $\delta$ have no dimension whereas $\beta$ and $1 / \gamma$ have the same dimension as $w^{ \pm}$. Both transformations map the $A d S_{3}$ boundary to itself and act on the boundary as the usual conformal transformations of $(1+1)$-dimensional Minkowski spacetime.

Let us consider a string configuration which extends straight from the AdS boundary at $z=0$ to the Poincare horizon at $z=\infty$ in the $z$ direction and moves with constant velocity $v$ in the $x$ direction

$$
x=v t, \quad t=\tau, \quad z=\sigma,
$$

which simply satisfies the string equation (2.4) as is seen in (2.11), and is dual to an isolated infinitely-massive quark moving with constant velocity $v$. We perform the $\mathrm{SL}(2, R)_{L}$ transformation with $\gamma \neq 0$ for the straight moving string solution to obtain an accelerating string configuration associated with proper acceleration $\gamma \sqrt{(1+v) /(1-v)}$ of a quark-antiquark pair in the following form

$$
x^{\prime}-\frac{1}{2 \gamma}\left(\alpha+\frac{1-v}{1+v} \delta\right)= \pm\left[\left(t^{\prime}-\frac{1}{2 \gamma}\left(\alpha-\frac{1-v}{1+v} \delta\right)\right)^{2}+\frac{1-v}{1+v} \frac{1}{\gamma^{2}}-z^{\prime 2}\right]^{1 / 2}
$$

where there are some constant shifts in $x^{\prime}$ and $t^{\prime}$ compared with (2.7). By making the replacement $(1-v) /(1+v) \gamma^{2} \rightarrow(1-v) /(1+v) \gamma^{2}+z_{m}^{2}$ for (3.4) we obtain the accelerating string solution associated with the finitely massive quark and antiquark and here write down explicitly the electric field $E$ exerted on the quark and antiquark from (2.10) as

$$
E=\frac{m}{(1-v) /(1+v) \gamma^{2}+\lambda / 4 \pi^{2} m^{2}} .
$$

Under a particular $\mathrm{SL}(2, R)_{L}$ transformation with $\gamma=0$ the string solutioin becomes

$$
x^{\prime}=v^{\prime} t^{\prime}+\frac{(1-v) \alpha \beta}{(1+v) \alpha^{2}+1-v} .
$$


with $v^{\prime}=\left((1+v) \alpha^{2}-(1-v)\right) /\left((1+v) \alpha^{2}+1-v\right)$. The transformed string solution shows a straight moving string with the different velocity $v^{\prime}$. The particular $\operatorname{SL}(2, R)_{L}$ transformation (3.1) with $\gamma=0$ is expressed as

$$
\left(\begin{array}{c}
t^{\prime} \\
x^{\prime}
\end{array}\right)=\frac{1}{2}\left(\begin{array}{cc}
\alpha^{2}+1 & \alpha^{2}-1 \\
\alpha^{2}-1 & \alpha^{2}+1
\end{array}\right)\left(\begin{array}{c}
t \\
x
\end{array}\right)+\frac{\alpha \beta}{2}, \quad z^{\prime}=\alpha z .
$$

Through the scaling $t^{\prime}=\alpha t^{\prime \prime}, x^{\prime}=\alpha x ", z^{\prime}=\alpha z^{\prime \prime}$ this transformation becomes

$$
\left(\begin{array}{c}
t " \\
x "
\end{array}\right)=\frac{1}{2 \alpha}\left(\begin{array}{cc}
\alpha^{2}+1 & \alpha^{2}-1 \\
\alpha^{2}-1 & \alpha^{2}+1
\end{array}\right)\left(\begin{array}{c}
t \\
x
\end{array}\right)+\frac{\beta}{2}, \quad \quad z^{\prime \prime}=z,
$$

which shows a combination of a Lorentz transformation and a translation in $(1+1)$ dimensional Minkowski spacetime at the AdS boundary.

Now we apply the $\mathrm{SL}(2, R)_{L}$ transformation to the accelerating string solution (2.7) which is figured out by the expanding semicircle $x^{2}+z^{2}=t^{2}+b^{2}$ for $0 \leq z$ in the $(x, z)$ plane. The first relation in (3.1) gives $x+t$ expressed in terms of $x^{\prime}+t^{\prime}$, which is substituted into the third relation in (3.1) to obtain $z$ expressed in terms of $x^{\prime}+t^{\prime}$ and $z^{\prime}$. We combine $x-t=\left(b^{2}-z^{2}\right) /(x+t)$ with the second relation in (3.1) to derive the third-order equation for $x^{\prime}$. The third-order equation becomes a product of a first-order one and a second-order one so that we have two curves

$$
\begin{aligned}
x^{\prime} & =-t^{\prime}+\frac{\alpha}{\gamma} \\
\left(x^{\prime}+\frac{b^{2} \gamma-\beta}{2 \delta}\right)^{2} & =\left(t^{\prime}-\frac{b^{2} \gamma+\beta}{2 \delta}\right)^{2}+\frac{b^{2}}{\delta^{2}}-z^{\prime 2} .
\end{aligned}
$$

The former (3.9) corresponds to (2.11) with $A=1$. Since $A$ is not allowed to be equated with 0,1 , it does not satisfy the string equation. The latter (3.10) shows the expanding string where the acceleration of a quark-antiquark pair changes from $1 / b$ to $\delta / b$. In a particular $\gamma=0$ case the transformed string configuration becomes a second-order equation for $x^{\prime}$ which is also given by (3.10) with $\gamma=0$. In a particular $\delta=0$ case the accelerating string solution is transformed to a product of two curves which represent a moving string solution with constant velocity

$$
x^{\prime}=\frac{\beta^{2}-b^{2}}{\beta^{2}+b^{2}} t^{\prime}-\frac{b^{2} \alpha \beta}{\beta^{2}+b^{2}}
$$

and the expression (3.9) which does not obey the string equation.

When the $\mathrm{SL}(2, R)_{R}$ transformation (3.2) is applied to the moving string with constant velocity (3.3) and the expanding string (2.7), the generally mapped string configurations are described by (3.4) with $t^{\prime}, v$ replaced by $-t^{\prime},-v$ and (3.9), (3.10) with $t^{\prime}$ replaced by $-t^{\prime}$ respectively.

Here we consider the special conformal transformation of the Poincare $A d S_{3}$ spacetime coordinates $z, x^{\mu}=(t, x)$

$$
x^{\mu \prime}=\frac{x^{\mu}+a^{\mu}\left(z^{2}+x^{2}\right)}{1+2 a \cdot x+a^{2}\left(z^{2}+x^{2}\right)}, \quad z^{\prime}=\frac{z}{1+2 a \cdot x+a^{2}\left(z^{2}+x^{2}\right)},
$$


which was studied for the circular Wilson loop [39]. We can analyze the special conformal transformation in the Poincare $A d S_{5}$ spacetime which is specified by (3.12) with $\mu=$ $0,1,2,3$, but for simplicity we restrict ourselves to the $A d S_{3}$ spacetime. The two cases with $a^{\mu}=(-a, a)$ and $a^{\mu}=(a, a)$ coincide with the particular $\mathrm{SL}(2, R)_{L}$ and $\mathrm{SL}(2, R)_{R}$ transformations with $\alpha=1, \beta=0, \gamma=2 a, \delta=1$ respectively.

Let us make a special conformal transformation with $a^{\mu}=\left(a^{0}, a^{1}\right)$ for the straight string moving with constant velocity $v(3.3)$ to have

$$
t^{\prime}=\frac{t+a^{0}\left(z^{2}-t^{2}+v^{2} t^{2}\right)}{P}, \quad x^{\prime}=\frac{v t+a^{1}\left(z^{2}-t^{2}+v^{2} t^{2}\right)}{P}, \quad z^{\prime}=\frac{z}{P}
$$

with

$$
P=1+2\left(-a^{0}+a^{1} v\right) t+\left(-\left(a^{0}\right)^{2}+\left(a^{1}\right)^{2}\right)\left(z^{2}-t^{2}+v^{2} t^{2}\right) .
$$

The first and second relations in (3.13) lead to $a^{1} t-a^{0} v t=\left(a^{1} t^{\prime}-a^{0} x^{\prime}\right) P$ which gives

$$
t=\frac{\left(a^{1} t^{\prime}-a^{0} x^{\prime}\right) P}{a^{1}-a^{0} v} .
$$

Substitution of (3.15) into (3.14) generates a second-order equation for $P$

$$
\left(-\left(a^{0}\right)^{2}+\left(a^{1}\right)^{2}\right) Q_{1} P^{2}+\left(Q_{2}-1\right) P+1=0
$$

with

$$
\begin{aligned}
& Q_{1}=z^{\prime 2}-\left(1-v^{2}\right)\left(\frac{a^{1} t^{\prime}-a^{0} x^{\prime}}{a^{1}-a^{0} v}\right)^{2}, \\
& Q_{2}=2 \frac{a^{1} v-a^{0}}{a^{1}-a^{0} v}\left(a^{1} t^{\prime}-a^{0} x^{\prime}\right) .
\end{aligned}
$$

We substitute (3.15), $z=P z^{\prime}$ and the solution of (3.16) into the second relation in (3.13) to obtain

$$
x^{\prime}=\frac{1}{2\left(a^{1}-a^{0} v\right)} \pm \sqrt{\left(t^{\prime}-\frac{v}{2\left(a^{1}-a^{0} v\right)}\right)^{2}+\frac{1-v^{2}}{4\left(a^{1}-a^{0} v\right)^{2}}-z^{\prime 2}}
$$

for $a^{1} \neq a^{0} v$. Thus we have the expanding string solution with acceleration $2 \mid a^{1}-$ $a^{0} v \mid / \sqrt{1-v^{2}}$. In a particular $a^{1}=a^{0} v$ case from the equation (3.15) the transformed string configuration is expressed as $x^{\prime}=\left(a^{1} / a^{0}\right) t^{\prime}=v t^{\prime}$ which is the moving string with the same velocity as the starting string.

For the expanding string (2.7) with acceleration $1 / b$ we perform a special conformal transformation with $a^{\mu}=\left(a^{0}, a^{1}\right)$ to obtain

$$
t^{\prime}=\frac{t+a^{0} b^{2}}{P}, \quad x^{\prime}=\frac{x+a^{1} b^{2}}{P}, \quad z^{\prime}=\frac{z}{P}
$$

with

$$
P=1+2\left(-a^{0} t+a^{1} x\right)+\left(-\left(a^{0}\right)^{2}+\left(a^{1}\right)^{2}\right) b^{2} .
$$


Combining the first and second relations in (3.19) with (3.20) we have

$$
P=\frac{1+\left(\left(a^{0}\right)^{2}-\left(a^{1}\right)^{2}\right) b^{2}}{1+2\left(a^{0} t^{\prime}-a^{1} x^{\prime}\right)} .
$$

Substituting three relations in (3.19) into (2.7) we have a second-order equation for $P$

$$
\left(z^{\prime 2}-t^{\prime 2}+x^{\prime 2}\right) P^{2}+2\left(a^{0} t^{\prime}-a^{1} x^{\prime}\right) b^{2} P+b^{4}\left(-\left(a^{0}\right)^{2}+\left(a^{1}\right)^{2}\right)-b^{2}=0 .
$$

Equating (3.21) with the solution of (3.22) leads to

$$
\begin{aligned}
\left(x^{\prime}+\frac{a^{1} b^{2}}{1+\left(\left(a^{0}\right)^{2}-\left(a^{1}\right)^{2}\right) b^{2}}\right)^{2}= & \left(t^{\prime}+\frac{a^{0} b^{2}}{1+\left(\left(a^{0}\right)^{2}-\left(a^{1}\right)^{2}\right) b^{2}}\right)^{2} \\
& +\frac{b^{2}}{\left[1+\left(\left(a^{0}\right)^{2}-\left(a^{1}\right)^{2}\right) b^{2}\right]^{2}}-z^{\prime 2}
\end{aligned}
$$

for $1+\left(\left(a^{0}\right)^{2}-\left(a^{1}\right)^{2}\right) b^{2} \neq 0$. Thus the magnitude of acceleration changes from $1 / b$ to $\left|1+\left(\left(a^{0}\right)^{2}-\left(a^{1}\right)^{2}\right) b^{2}\right| / b$. In a particular case $1+\left(\left(a^{0}\right)^{2}-\left(a^{1}\right)^{2}\right) b^{2}=0$ with $\left(a^{1}\right)^{2}>$ $\left(a^{0}\right)^{2} \neq 0$ the expanding string turns back to a moving string with constant velocity $x^{\prime}=\left(a^{0} / a^{1}\right) t^{\prime}+1 / 2 a^{1}$, which is derived from (3.21). Further in the $a^{\mu}=(0, \pm 1 / b)$ cases the expansion string becomes a static straight string located at $x^{\prime}= \pm b / 2$ stretching from the AdS boundary to the Poincare horizon, which is dual to a static isolated quark.

Here we restore the AdS radius $R$ to express the following relations between the Poincare coordinates in $A d S_{3}$ and the embedding coordinates $X^{M}(M=-1,0,1,2)$ on which the conformal $\mathrm{SO}(2,2)$ transformation is acting linearly

$$
\begin{aligned}
X^{\mu} & =\frac{x^{\mu}}{z} R,(\mu=0,1), \\
X^{-1} & =\frac{R^{2}+z^{2}+x_{\mu} x^{\mu}}{2 z}, \quad X^{2}=\frac{R^{2}-z^{2}-x_{\mu} x^{\mu}}{2 z}, \\
-R^{2} & =-\left(X^{-1}\right)^{2}-\left(X^{0}\right)^{2}+\left(X^{1}\right)^{2}+\left(X^{2}\right)^{2} .
\end{aligned}
$$

Although we can analyze the conformal $\mathrm{SO}(2,4)$ transformation acting on the embedding coordinates $X^{M}(M=-1,0,1,2,3,4)$ for $A d S_{5}$, for simplicity we consider the conformal $\mathrm{SO}(2,2)$ transformation.

For the moving string with constant velocity $v$, which is described by $X^{1}=v X^{0}$, we perform one conformal $\mathrm{SO}(2,2)$ transformation

$$
X^{-1^{\prime}}=-X^{0}, \quad X^{0^{\prime}}=X^{-1}, X^{1^{\prime}}=X^{1}, X^{2^{\prime}}=X^{2},
$$

which interchanges $X^{-1}$ and $X^{0}$. The transformed configuration is specified by $X^{1^{\prime}}=$ $-v X^{-1^{\prime}}$ that is expressed in terms of the Poincare coordinates as

$$
\left(x^{\prime}+\frac{R}{v}\right)^{2}=t^{\prime 2}+\frac{1-v^{2}}{v^{2}} R^{2}-z^{\prime 2},
$$

which represents the expanding string with acceleration $v / R \sqrt{1-v^{2}}$. 
The other conformal $\mathrm{SO}(2,2)$ transformation defined as the interchange between $X^{1}$ and $X^{2}$

$$
X^{-1^{\prime}}=X^{-1}, \quad X^{0^{\prime}}=X^{0}, \quad X^{1^{\prime}}=-X^{2}, \quad X^{2^{\prime}}=X^{1}
$$

produces $X^{2^{\prime}}=v X^{0^{\prime}}$ which becomes

$$
x^{\prime 2}=\left(t^{\prime}-v R\right)^{2}+\left(1-v^{2}\right) R^{2}-z^{\prime 2} .
$$

Thus the expanding string solution with acceleration $1 / R \sqrt{1-v^{2}}$ is constructed.

Now performing the conformal $\mathrm{SO}(2,2)$ transformation (3.25) for the expanding string (2.7) which is expressed as

$$
\left(X^{0}\right)^{2}-\left(X^{1}\right)^{2}=R^{2}-\frac{b^{2}}{R^{2}}\left(X^{-1}+X^{2}\right)^{2}
$$

we obtain a curve

$$
\left(X^{-1^{\prime}}\right)^{2}-\left(X^{1^{\prime}}\right)^{2}=R^{2}-\frac{b^{2}}{R^{2}}\left(X^{0^{\prime}}+X^{2^{\prime}}\right)^{2}
$$

The mapped expression is a polynomial of $x^{\prime}$ in the fourth order which is compared with the second-order plynomial of $x$ in (3.29). It, however, is expressed in terms of $y \equiv x^{\prime 2}+z^{\prime 2}$ as

$$
\begin{aligned}
\left(1+\frac{b^{2}}{R^{2}}\right) y^{2}-2 & \left(t^{\prime 2}+R^{2}+\frac{b^{2}}{R^{2}}\left(t^{\prime}+R\right)^{2}\right) y \\
& +\left(t^{\prime 2}-R^{2}\right)^{2}+\frac{b^{2}}{R^{2}}\left(t^{\prime}+R\right)^{4}=0
\end{aligned}
$$

so that we have two curves

$$
\begin{aligned}
& x^{\prime 2}=\left(t^{\prime}+R\right)^{2}-z^{\prime 2}, \\
& x^{\prime 2}=\left(t^{\prime}+\frac{b^{2}-R^{2}}{b^{2}+R^{2}} R\right)^{2}+\frac{4 b^{2} R^{4}}{\left(b^{2}+R^{2}\right)^{2}}-z^{\prime 2} .
\end{aligned}
$$

The former (3.32) corresponds to $(2.7)$ with $b=0$. Since $B$ is not allowed to be 0 , it does not solve the string equation. The latter (3.33) is the expanding string solution with acceleration $\left(b^{2}+R^{2}\right) / 2 b R^{2}$.

The other conformal $\mathrm{SO}(2,2)$ transformation (3.27) applied to the expanding string solution (3.29) produces a curve

$$
\left(X^{0^{\prime}}\right)^{2}-\left(X^{2^{\prime}}\right)^{2}=R^{2}-\frac{b^{2}}{R^{2}}\left(X^{-1^{\prime}}-X^{1^{\prime}}\right)^{2},
$$

which is similarly expressed in terms of $y \equiv t^{\prime 2}-z^{\prime 2}$ as

$$
\begin{aligned}
\left(1-\frac{b^{2}}{R^{2}}\right) y^{2}-2 & \left(x^{\prime 2}+R^{2}-\frac{b^{2}}{R^{2}}\left(x^{\prime}-R\right)^{2}\right) y \\
& +\left(x^{\prime 2}-R^{2}\right)^{2}-\frac{b^{2}}{R^{2}}\left(x^{\prime}-R\right)^{4}=0 .
\end{aligned}
$$


For $R \neq b$ two curves are obtained by

$$
\begin{aligned}
& t^{\prime 2}-z^{\prime 2}=\left(x^{\prime}-R\right)^{2} \\
& t^{\prime 2}-z^{\prime 2}=\frac{1}{1-b^{2} / R^{2}}\left(\left(x^{\prime}+R\right)^{2}-\frac{b^{2}}{R^{2}}\left(x^{\prime}-R\right)^{2}\right) .
\end{aligned}
$$

The former (3.36) also does not obey the string equation, while the latter (3.37) yields the expanding string solution with acceleratioin $\left|b^{2}-R^{2}\right| / 2 b R^{2}$ as shown by

$$
\left(x^{\prime}-\frac{b^{2}+R^{2}}{b^{2}-R^{2}} R\right)^{2}=t^{\prime 2}+\frac{4 b^{2} R^{4}}{\left(b^{2}-R^{2}\right)^{2}}-z^{\prime 2} .
$$

In a particular $R=b$ case the equation (3.35) leads to

$$
x^{\prime}\left(\left(x^{\prime}-R\right)^{2}-\left(t^{2}-z^{\prime 2}\right)\right)=0,
$$

which gives two curves $x^{\prime}=0$ and $x^{\prime}=R \pm \sqrt{t^{\prime 2}-z^{\prime 2}}$. The former shows the static string solution, while the latter does not satisfy the string equation.

\section{The accelerating string in the string sigma model action}

Let us consider a time-dependent open string configuration in $A d S_{3}$ with the Poincare metric by analyzing the string sigma model action in the Lorentzian worldsheet coordinates with $a=0,1$

$$
S=-\frac{\sqrt{\lambda}}{4 \pi} \int d \tau d \sigma \frac{1}{z^{2}}\left(-\partial_{a} t \partial^{a} t+\partial_{a} x \partial^{a} x+\partial_{a} z \partial^{a} z\right) .
$$

The off-diagonal Virasoro constraint gives

$$
-\dot{t} t^{\prime}+\dot{x} x^{\prime}+\dot{z} z^{\prime}=0
$$

In this section we use the dot and prime as the derivatives with respect to $\tau$ and $\sigma$ respectively.

Here we choose the following ansatz in the factorized form

$$
t=t_{\tau}(\tau) f(\sigma), \quad x=x_{\tau}(\tau) f(\sigma), \quad z=z(\sigma) .
$$

The off-diagonal Virasoro constraint (4.2) reads

$$
-\dot{t_{\tau}} t_{\tau}+\dot{x_{\tau}} x_{\tau}=0
$$

which is solved by

$$
x_{\tau}^{2}-t_{\tau}^{2}= \pm N^{2}
$$

with an integration constant $N$.

First we consider the plus case to parameterize $x_{\tau}$ and $t_{\tau}$ in terms of a positive parameter $p$ as

$$
x_{\tau}=N \cosh p \tau, \quad t_{\tau}=N \sinh p \tau
$$


with $-\infty<\tau<\infty$. The integration constant $N$ is absorbed into $f(\sigma)$ so that $N$ can be set to unity.

The diagonal Virasoro constraint gives

$$
f^{\prime 2}-p^{2} f^{2}+z^{\prime 2}=0
$$

Substituting the ansatz (4.3) with (4.6) into the equations of motion for $t$ and $x$

$$
\partial_{\tau}\left(\frac{\dot{t}}{z^{2}}\right)-\partial_{\sigma}\left(\frac{t^{\prime}}{z^{2}}\right)=0, \partial_{\tau}\left(\frac{\dot{x}}{z^{2}}\right)-\partial_{\sigma}\left(\frac{x^{\prime}}{z^{2}}\right)=0
$$

we have an identical equation

$$
f^{\prime \prime}=\frac{2 z^{\prime}}{z} f^{\prime}+p^{2} f
$$

The equation of motion for $z$

$$
\partial_{\tau}\left(\frac{\dot{z}}{z^{2}}\right)-\partial_{\sigma}\left(\frac{z^{\prime}}{z^{2}}\right)=\frac{1}{z^{3}}\left(\left(\partial_{a} z\right)^{2}-\left(\partial_{a} t\right)^{2}+\left(\partial_{a} x\right)^{2}\right)
$$

turns out to be

$$
z z^{\prime \prime}=z^{\prime 2}-f^{\prime 2}-p^{2} f^{2}
$$

We sum (4.11) and (4.9) multiplied by $f$ to derive a differential equation

$$
\partial_{\sigma}^{2}\left(z^{2}+f^{2}\right)=\frac{2 z^{\prime}}{z} \partial_{\sigma}\left(z^{2}+f^{2}\right) .
$$

Here we consider a simple solution

$$
z^{2}+f^{2}=b^{2}
$$

with a constant positive parameter $b$, which implies $0 \leq z \leq b$. We substitute $f=$ $\pm \sqrt{b^{2}-z^{2}}$ of (4.13) into (4.7) to obtain an equation for $z$

$$
b^{2} z^{\prime 2}-p^{2}\left(b^{2}-z^{2}\right)^{2}=0 .
$$

Owing to $0 \leq z \leq b$ the solution of (4.14) is expressed as

$$
z=b \tanh p \sigma
$$

where we take the range of $\sigma$ as $0 \leq \sigma<\infty$. In the region $0 \leq z \leq b$ we have a string profile expressed by (4.15) and

$$
x= \pm \frac{b \cosh p \tau}{\cosh p \sigma}, \quad t= \pm \frac{b \sinh p \tau}{\cosh p \sigma} .
$$

Hereafter the plus and minus solutions are called by the I and II solutions respectively. These I and II solutions are confirmed to satisfy the string equations (4.8) and (4.10). Eliminating the dependences of the worldsheet coordinates we reproduce the accelerating string solution (2.7) for the region $0 \leq z \leq b$. 
Alternatively we replace $\sigma$ by $z$ through (4.15) to have

$$
x= \pm \sqrt{b^{2}-z^{2}} \cosh p \tau, \quad t= \pm \sqrt{b^{2}-z^{2}} \sinh p \tau .
$$

If we choose $p=1 / b$, then in the AdS boundary $z=0$, that is, $\sigma=0$ the solutions (4.17) become $x= \pm b \cosh \tau / b$ and $t= \pm b \sinh \tau / b$, which yield $x= \pm \sqrt{t^{2}+b^{2}}$ and represent the accelerating quark and antiquark trajectories for plus and minus signs respectively with proper time $\tau$ and proper acceleration $1 / b$. The expressions of (4.15) and (4.17) with $p=1 / b$ agree with ones in ref. [11] which are described as a static solution in the generalized Rindler spacetime that is derived from the AdS spacetime by a coordinate transformation. The string in the generalized Rindler spacetime was analyzed in $[18,22]$ where the thermodynamics associated with the worldsheet horizon which has the Unruh temperature is further studied.

For fixed $\sigma$, that is, fixed $z$ in the I solution the limit $\tau=-\infty$ leads to $t=-\infty, x=\infty$ and $\tau=0$ gives $t=0, x=b / \cosh (\sigma / b)=\sqrt{b^{2}-z^{2}}$ that is the position of the string bit at depth $z$ and at time $t=0$. The limit $\tau=\infty$ leads to $t=\infty, x=\infty$, and there is a restriction $|t|<x$ for each $\tau$ through $t / x=\tanh \tau / b$. On the other hand in the II solution, $\tau=\infty$ corresponds to $t=-\infty, x=-\infty, \tau=0$ to $t=0, x=-\sqrt{b^{2}-z^{2}}$ and $\tau=-\infty$ to $t=\infty, x=-\infty$, which imply $x<-|t|$ for each $\tau$.

For fixed $t$ the I string extends from the quark location $(x, z)=\left(\sqrt{t^{2}+b^{2}}, 0\right)$ specified by $\sigma=0$ to $(x, z)=(|t|, b)$ in an arc, while the II string extends from the antiquark location $(x, z)=\left(-\sqrt{t^{2}+b^{2}}, 0\right)$ to $(x, z)=(-|t|, b)$ similarly.

Here let us consider the minus case for (4.5) with $N=1$ and represent $x_{\tau}$ and $t_{\tau}$ as

$$
x_{\tau}=\sinh p \tau, \quad t_{\tau}=\cosh p \tau .
$$

The diagonal Virasoro constraint yields

$$
-f^{\prime 2}+p^{2} f^{2}+z^{\prime 2}=0 .
$$

In this case the equations of motion for $t$ and $x$ lead to the same equation as (4.9), however, the equation of motion for $z$ gives

$$
z z^{\prime \prime}=z^{\prime 2}+f^{\prime 2}+p^{2} f^{2} .
$$

Combining together we derive

$$
\partial_{\sigma}^{2}\left(z^{2}-f^{2}\right)=\frac{2 z^{\prime}}{z} \partial_{\sigma}\left(z^{2}-f^{2}\right),
$$

which has two simple solutions $z^{2}-f^{2}= \pm b^{2}$, where the upper sign case has a restriction $b \leq z$. The equation (4.19) with $f^{2}=z^{2} \mp b^{2}$ can be expressed as

$$
\mp \frac{b^{2}{z^{\prime}}^{2}}{z^{2} \mp b^{2}}+p^{2}\left(z^{2} \mp b^{2}\right)=0 .
$$

This equation of $z$ for the lower sign has no real solution, while for the upper sign it has the following solution

$$
z=\frac{b}{\tanh p \sigma}
$$


which yields

$$
x= \pm \frac{b \sinh p \tau}{\sinh p \sigma}, \quad t= \pm \frac{b \cosh p \tau}{\sinh p \sigma}
$$

with $0 \leq \sigma<\infty,-\infty<\tau<\infty$. To the plus and minus solutions we call the III and IV solutions. These solutions are expressed as

$$
x= \pm \sqrt{z^{2}-b^{2}} \sinh p \tau, \quad t= \pm \sqrt{z^{2}-b^{2}} \cosh p \tau,
$$

which also reproduce the accelerating string solution (2.7) through the elimination of $\tau$ for the region $b \leq z \leq \sqrt{t^{2}+b^{2}}$.

In the IV solution of (4.24) and (4.25) with $p=1 / b$ for fixed $\tau, t$ is negative and changes from $t=-\infty$ at $\sigma=0$ to $t=0$ at $\sigma=\infty$, where the roles of $\tau$ and $\sigma$ are exchanged in comparison with the I and II solutions. Owing to $x / t=\tanh \tau / b, x$ varies such that $x=t=-|t|$ at $\tau=\infty, x=0$ at $\tau=0$ and $x=-t=|t|$ at $\tau=-\infty$. At fixed $t<0$ the IV string extends from $(x, z)=(-|t|, b)$ to $(x, z)=(|t|, b)$ in an arc and shrinks to zero at $t=0$

On the other hand in the III solution for fixed $\tau, t$ is positive and changes from $t=0$ at $\sigma=\infty$ to $t=\infty$ at $\sigma=0$. Owing to $x / t=\tanh \tau / b, x$ varies such that $x=-t$ at $\tau=-\infty, x=0$ at $\tau=0$ and $x=t$ at $\tau=\infty$. At fixed $t, z$ is described by $z=b\left(1+t^{2} /(b \cosh \tau / b)^{2}\right)^{1 / 2}$ so that $z$ becomes $z=b$ at $\tau= \pm \infty$ that implies $\sigma=\infty$ for $t$ to be fixed, and $z=\sqrt{t^{2}+b^{2}}$ at $\tau=0$. Thus at $t=0$ the III string starts as a point at $(x, z)=(0, b)$ and at fixed $t>0$ extends from $(x, z)=(-t, b)$ to $(x, z)=(t, b)$ through $(x, z)=\left(0, \sqrt{t^{2}+b^{2}}\right)=(0, b / \tanh \sigma / b)$.

Now we calculate the induced metric on the string surface, (4.15) and (4.16) in the region $0 \leq z \leq b$ to obtain a conformally flat expression

$$
d s_{w s}^{2}=\frac{1}{b^{2} \sinh ^{2}(\sigma / b)}\left(-d \tau^{2}+d \sigma^{2}\right)
$$

which has a horizon at $\sigma=\infty$ on the worldsheet that yields $z=b$ as a dividing line in the bulk spacetime. The induced metric on the string surface, (4.23) and (4.24) in the region $b \leq z$ is described by a different expression

$$
d s_{w s}^{2}=\frac{1}{b^{2} \cosh ^{2}(\sigma / b)}\left(d \tau^{2}-d \sigma^{2}\right),
$$

which has also a horizon at $\sigma=\infty$ on the worldsheet that corresponds to $z=b$.

If we make a coordinate transformation from $\sigma$ to $z$ using (4.15) and (4.23) to rewrite (4.26) and (4.27) in terms of $\tau$ and $z$ respectively, we obtain a single expression

$$
d s_{w s}^{2}=\frac{1}{z^{2}}\left(-\left(1-\frac{z^{2}}{b^{2}}\right) d \tau^{2}+\frac{d z^{2}}{1-z^{2} / b^{2}}\right)
$$

where there is a horizon at $z=b$. In the interior region $b \leq z$ the roles of $\tau$ and $z$ are exchanged such that $\tau$ becomes a spacelike coordinate and $z$ becomes a timelike coordinate, which corresponds to the exchange of the roles of $\tau$ and $\sigma$ between (4.26) and (4.27). 
Combining the above discussed behaviors of the string solutions I, II, III and IV derived from the string sigma model action, we have the following picture. In the early time specified by $t<0$, the right string I and the left string II staying in the exterior region $0 \leq$ $z \leq b$ are decelerated and connected at $z=b$ by the middle string IV staying in the interior region $b \leq z \leq \sqrt{t^{2}+b^{2}}$. The connected string shape is expressed as $x^{2}=t^{2}+b^{2}-z^{2}$. At $t=0$ the interior string IV shrinks to zero and the two exterior strings I and II stop and directly touch at $z=b$ where the connected string shape is expressed as $x^{2}=b^{2}-z^{2}$. In the late time $t>0$, the exterior strings I and II return back and are accelerated in opposite directions, where the two exterior strings are connected by the interior string III. Thus in the early time $t<0$ the string IV is interpreted as a wormhole connecting two separated exterior regions, while in the late time $t>0$ the wormhole is represented by the string III.

\section{Conclusion}

For the open string in the Poincare $A d S_{3}$ spacetime we have used the Nambu-Goto action in the static gauge to make an ansatz in a square root expression characterized by three parameters for the string profile. We have observed that if three parameters are appropriately chosen, there appear two open string solutions in a complementary pair, the string solution associated with the one-cusp Wilson loop [33] and the expanding string solution associated with a uniformly accelerating quark-antiquark pair [11].

We have constructed the expanding string solution by applying the $\mathrm{SL}(2, R)_{L} \times$ $\mathrm{SL}(2, R)_{R}$ isometry transfomations, the special conformal transformations and the conformal $\mathrm{SO}(2,2)$ transformations to a simple moving string solution dual to a moving quark with constant velocity. We have demonstrated that under the three kinds of transformations the expanding string solution is usually mapped to the same expanding string with the different acceleration. It has been observed that some particular transformations make the expanding string solution change back to the moving string solution with constant velocity or the static string solution.

Based on the string sigma model action we have made an ansatz for the open string profile in the factorized form and constructed two kinds of string solutions, the exteror strings and the interior strings that stay in the two different bulk spacetime regions. We have observed that on each string worldsheet there appear the horizon which is associated with the dividing line which separates the two different bulk spacetime regions. We have demonstrated that the expanding string solution is constructed by connecting two separated exterior strings with one interior string.

Open Access. This article is distributed under the terms of the Creative Commons Attribution License (CC-BY 4.0), which permits any use, distribution and reproduction in any medium, provided the original author(s) and source are credited.

\section{References}

[1] J.M. Maldacena, The large- $N$ limit of superconformal field theories and supergravity, Adv. Theor. Math. Phys. 2 (1998) 231 [hep-th/9711200] [INSPIRE]. 
[2] S.S. Gubser, I.R. Klebanov and A.M. Polyakov, Gauge theory correlators from non-critical string theory, Phys. Lett. B 428 (1998) 105 [hep-th/9802109] [InSPIRE].

[3] E. Witten, Anti-de Sitter space and holography, Adv. Theor. Math. Phys. 2 (1998) 253 [hep-th/9802150] [INSPIRE].

[4] C.P. Herzog, A. Karch, P. Kovtun, C. Kozcaz and L.G. Yaffe, Energy loss of a heavy quark moving through $N=4$ supersymmetric Yang-Mills plasma, JHEP 07 (2006) 013 [hep-th/0605158] [INSPIRE].

[5] S.S. Gubser, Drag force in AdS/CFT, Phys. Rev. D 74 (2006) 126005 [hep-th/0605182] [INSPIRE].

[6] A. Karch and E. Katz, Adding flavor to AdS/CFT, JHEP 06 (2002) 043 [hep-th/0205236] [INSPIRE].

[7] A. Mikhailov, Nonlinear waves in AdS/CFT correspondence, hep-th/0305196 [INSPIRE].

[8] M. Chernicoff and A. Güijosa, Acceleration, Energy Loss and Screening in Strongly-Coupled Gauge Theories, JHEP 06 (2008) 005 [arXiv:0803.3070] [INSPIRE].

[9] M. Chernicoff, J.A. García and A. Güijosa, Generalized Lorentz-Dirac Equation for a Strongly-Coupled Gauge Theory, Phys. Rev. Lett. 102 (2009) 241601 [arXiv:0903.2047] [INSPIRE].

[10] M. Chernicoff, J.A. García and A. Güijosa, A Tail of a Quark in $N=4 S Y M$, JHEP 09 (2009) 080 [arXiv:0906.1592] [INSPIRE].

[11] B.-W. Xiao, On the exact solution of the accelerating string in $A d S_{5}$ space, Phys. Lett. B 665 (2008) 173 [arXiv:0804.1343] [INSPIRE].

[12] A. Paredes, K. Peeters and M. Zamaklar, Temperature versus acceleration: The Unruh effect for holographic models, JHEP 04 (2009) 015 [arXiv:0812.0981] [INSPIRE].

[13] S.S. Gubser, Momentum fluctuations of heavy quarks in the gauge-string duality, Nucl. Phys. B 790 (2008) 175 [hep-th/0612143] [INSPIRE].

[14] J. Casalderrey-Solana and D. Teaney, Transverse Momentum Broadening of a Fast Quark in a $\mathcal{N}=4$ Yang-Mills Plasma, JHEP 04 (2007) 039 [hep-th/0701123] [INSPIRE].

[15] G. Beuf, C. Marquet and B.-W. Xiao, Heavy-quark energy loss and thermalization in a strongly coupled SYM plasma, Phys. Rev. D 80 (2009) 085001 [arXiv:0812.1051] [INSPIRE].

[16] J. de Boer, V.E. Hubeny, M. Rangamani and M. Shigemori, Brownian motion in AdS/CFT, JHEP 07 (2009) 094 [arXiv: 0812.5112] [INSPIRE].

[17] D.T. Son and D. Teaney, Thermal Noise and Stochastic Strings in AdS/CFT, JHEP 07 (2009) 021 [arXiv:0901.2338] [INSPIRE].

[18] E. Cáceres, M. Chernicoff, A. Güijosa and J.F. Pedraza, Quantum Fluctuations and the Unruh Effect in Strongly-Coupled Conformal Field Theories, JHEP 06 (2010) 078 [arXiv: 1003.5332] [INSPIRE].

[19] J.A. García, A. Güijosa and E.J. Pulido, No Line on the Horizon: On Uniform Acceleration and Gluonic Fields at Strong Coupling, JHEP 01 (2013) 096 [arXiv:1210.4175] [INSPIRE].

[20] A. Güijosa and J.F. Pedraza, Early-Time Energy Loss in a Strongly-Coupled SYM Plasma, JHEP 05 (2011) 108 [arXiv:1102 .4893] [INSPIRE]. 
[21] M. Chernicoff and A. Paredes, Accelerated detectors and worldsheet horizons in AdS/CFT, JHEP 03 (2011) 063 [arXiv: 1011.4206] [INSPIRE].

[22] T. Hirayama, P.-W. Kao, S. Kawamoto and F.-L. Lin, Unruh effect and Holography, Nucl. Phys. B 844 (2011) 1 [arXiv: 1001.1289] [INSPIRE].

[23] V.E. Hubeny and G.W. Semenoff, String worldsheet for accelerating quark, arXiv:1410.1171 [INSPIRE].

[24] B. Fiol, A. Güijosa and J.F. Pedraza, Branes from Light: Embeddings and Energetics for Symmetric k-Quarks in $\mathcal{N}=4$ SYM, JHEP 01 (2015) 149 [arXiv:1410.0692] [INSPIRE].

[25] J. Maldacena and L. Susskind, Cool horizons for entangled black holes, Fortsch. Phys. 61 (2013) 781 [arXiv:1306. 0533] [INSPIRE].

[26] K. Jensen and A. Karch, The holographic dual of an EPR pair has a wormhole, Phys. Rev. Lett. 111 (2013) 211602 [arXiv:1307.1132] [INSPIRE].

[27] J. Sonner, Holographic Schwinger effect and the geometry of entanglement, Phys. Rev. Lett. 111 (2013) 211603 [arXiv:1307.6850] [INSPIRE].

[28] M. Chernicoff, A. Güijosa and J.F. Pedraza, Holographic EPR pairs, wormholes and radiation, JHEP 10 (2013) 211 [arXiv:1308.3695] [INSPIRE].

[29] K. Jensen, A. Karch and B. Robinson, The holographic dual of a Hawking pair has a wormhole, Phys. Rev. D 90 (2014) 064019 [arXiv: 1405. 2065] [INSPIRE].

[30] A. Lewkowycz and J. Maldacena, Exact results for the entanglement entropy and the energy radiated by a quark, JHEP 05 (2014) 025 [arXiv: 1312.5682] [INSPIRE].

[31] V.E. Hubeny and G.W. Semenoff, Holographic Accelerated Heavy Quark-Anti-Quark Pair, arXiv: 1410.1172 [INSPIRE].

[32] S. Seki and S.-J. Sin, EPR $=E R$ and scattering amplitude as entanglement entropy change, Phys. Lett. B 735 (2014) 272 [arXiv:1404.0794] [INSPIRE].

[33] M. Kruczenski, A Note on twist two operators in $\mathcal{N}=4 S Y M$ and Wilson loops in Minkowski signature, JHEP 12 (2002) 024 [hep-th/0210115] [INSPIRE].

[34] J.M. Maldacena and A. Strominger, AdS $S_{3}$ black holes and a stringy exclusion principle, JHEP 12 (1998) 005 [hep-th/9804085] [INSPIRE].

[35] M. Bañados, M. Henneaux, C. Teitelboim and J. Zanelli, Geometry of the $2+1$ black hole, Phys. Rev. D 48 (1993) 1506 [gr-qc/9302012] [inSPIRE].

[36] L.F. Alday and J.M. Maldacena, Gluon scattering amplitudes at strong coupling, JHEP 06 (2007) 064 [arXiv:0705.0303] [InSPIRE].

[37] S. Ryang, Conformal SO(2,4) Transformations of the One-Cusp Wilson Loop Surface, Phys. Lett. B 659 (2008) 894 [arXiv:0710.1673] [INSPIRE].

[38] S. Ryang, Nonstatic Ad $S_{2}$ branes and the isometry group of $A d S_{3}$ space-time, Mod. Phys. Lett. A 17 (2002) 309 [hep-th/0110008] [INSPIRE].

[39] D.E. Berenstein, R. Corrado, W. Fischler and J.M. Maldacena, The operator product expansion for Wilson loops and surfaces in the large-N limit, Phys. Rev. D 59 (1999) 105023 [hep-th/9809188] [INSPIRE]. 